\title{
The Excitation of the Far Ultraviolet Electroglow Emissions on Uranus, Saturn, and Jupiter
}

\author{
JOHN T. Clarke ${ }^{1}$ \\ Laboratory for Astronomy and Solar Physics, NASA Goddard Space Flight Center, Greenbelt, Maryland
}

MARY K. HUdSON

Department of Physics and Astronomy, Dartmouth College, Hanover, New Hampshire

YUK L. YUNG

Division of Geologic and Planetary Sciences, California Institute of Technology, Pasadena

\begin{abstract}
We propose that the diffuse FUV emissions of $\mathrm{H}$ and $\mathrm{H}_{2}$ in excess of photoelectron excitation observed from the sunlit atmospheres of Uranus, Saturn, and Jupiter are produced by electric field acceleration of photoelectrons and ions locally in the upper atmospheres. This in situ acceleration is required to satisfy the many observational constraints on the altitude distribution, exciting particle energy, and total input energy requirements of the electroglow mechanism. We further suggest that a primary mechanism leading to this acceleration is an ionospheric dynamo, which is created in the same manner as the Earth's dynamo. The calculated altitude of charge separation by the neutral wind drag on ions across magnetic field lines is consistent with the observed peaks in electroglow emissions from the Voyager ultraviolet spectrometer limb scan data on both Saturn (near the homopause) and Uranus (just above the homopause). This dynamo action therefore appears to initiate the acceleration process, which must have the form of field-aligned potentials to accelerate the magnetized electrons. We propose that these field-aligned potentials are due to anomalous resistivity, which results from sufficiently high fieldaligned currents in the ionosphere to generate plasma instabilities and therefore runaway electrons and ions above some critical lower initial energy. There are multiple candidate processes for inducing these currents, including polarization in the equivalent $F$ regions and inner magnetospheric convection, and each of these processes should exhibit latitudinal structure. The acceleration of low-energy electrons in an $\mathbf{H}_{2}$ atmosphere preferentially results in FUV radiation and further ionization, whereas electron acceleration in a nitrogen/oxygen atmosphere such as the Earth's is dominated by elastic scattering and thus results in electric currents. Individual electron and proton collisions with $\mathbf{H}_{2}$ molecules will result in excitation, ionization, and heating, so that considerable enhancement of the ionospheric density and heating of the upper atmosphere will accompany the FUV emission.
\end{abstract}

\section{INTRODUCTION}

The interpretation of the $\mathrm{H}$ and $\mathrm{H}_{2}$ FUV emissions observed from the upper atmospheres of the outer planets has followed an interesting (and evolving) course over the period of time since the launch of the International Ultraviolet Explorer (IUE) in January 1978, which covers the Voyager encounters with Jupiter, Saturn, and Uranus. Initially, interpretations of the $\mathrm{H} \mathrm{Ly} \alpha$ line emission from Jupiter and Saturn were all in terms of resonantly scattered solar radiation, and charged particle-excited emissions of $\mathrm{H}_{2}$ bands and $\mathrm{H}$ Ly $\alpha$ were believed to be confined to the bright polar aurora on both planets. It became apparent, however, that more diffuse $\mathrm{H}_{2}$ band emissions were present on both planets [Broadfoot et al., 1979, 1981; Clarke et al., 1982] at wavelengths where the solar flux for scattering by $\mathrm{H}_{2}$ is minimal, implying particle excitation of the $\mathrm{H}_{2}$. In addition, Clarke et al. [1979], in an observation made by a sounding rocket telescope, first showed that Jupiter displays a magnetically confined equatorial H Ly $\alpha$ bright region, subsequently known as the $\mathrm{H}$ Ly $\alpha$ bulge and confirmed by Voyager [Sandel et al., 1980]. These additional emissions were initially interpreted as being due to soft pre-

\footnotetext{
${ }^{1}$ Now at Department of Atmospheric and Oceanic Sciences, University of Michigan, Ann Arbor.

Copyright 1987 by the American Geophysical Union.
}

Paper number 7A9027.

0148-0227/87/007A-9027\$05.00 cipitating particles and/or an enhanced reflectivity to solar $\mathbf{H}$ Ly $\alpha$, with at most a partial contribution from photoelectrons. The idea of precipitating soft electrons seems to have been first independently proposed by Hunten and Dessler [1977] to explain the high thermospheric temperature of Jupiter observed by Pioneer 10 and 11 . However, the mechanism for producing these electrons was not investigated.

Following more detailed analyses of the Voyager ultraviolet spectrometer (UVS) spectra of Jupiter [Shemansky, 1985] and Saturn [Yelle et al., 1986], it became apparent that this diffuse emission is largely restricted to the sunlit atmosphere and that it is generated by soft $(10-50 \mathrm{eV})$ particle excitation with a peak near or just above the homopause in altitude and extending through the higher atmosphere. The observations by IUE [Clarke et al., 1986] and then by the UVS [Broadfoot et al., 1986] of an unexpectedly high brightness in these emissions from Uranus reemphasized the important clue that the radiated energy is greater than the solar EUV (i.e., photoelectron) energy input into the atmosphere. Although photoelectrons can act as a trigger for the process (which decays rapidly in the absence of sunlight), the energy generation must occur locally in the atmosphere. This process for producing both $\mathbf{H}$ Ly $\alpha$ and $\mathrm{H}_{2}$ emissions was named "electroglow" by Broadfoot et al. [1986].

In this paper we will briefly summarize the observational characteristics of the previously unexplained diffuse emissions, outline our chain of logic in deducing the nature of the excitation process, and discuss how the dynamo action of the 
ionosphere might lead to the required excitation. It is important to keep in mind that some of this emission is certainly produced by direct photoelectron excitation and that the theory developed here is to explain the additional energy source. Because of the many similarities between the diffuse emissions from the different planets, we assume that there must be one mechanism which applies to the outer planets in general, and we accept this as an additional constraint on our theory.

\section{Observational Summary}

\section{Jupiter}

The sunlit atmosphere produces copious amounts of $\mathrm{H} \mathrm{Ly} \propto$ emission, measured at the subsolar point to be 8-15 kR by IUE [Skinner et al., 1983, personal communication, 1987] and 15-20 kR by the UVS [Shemansky, 1985]. Nearly simultaneous observations during the Voyager encounters by the UVS and IUE indicate that the difference in measured brightness is due to calibration differences rather than real variability. The sunlit atmosphere $\mathrm{H}$ Ly $\alpha$ displays limb darkening by roughly a cosine function, indicating optically thick resonant scattering of solar emission. There is sufficient solar $\mathbf{H ~ L y}$ $\alpha$ at Jupiter to explain all of the observed emission by resonant scattering, and this makes it difficult to separate the contributions by diffuse particle excitation [Yung and Strobel, 1980]. However, there is a superimposed equatorial excess (the H Ly $\alpha$ bulge) above the modeled spatial distribution of the resonant scattered component at all longitudes [Clarke et al., 1981a] and peaking near $\lambda_{\text {III }}=100^{\circ}$. On the dark hemisphere the intensity is $800 \mathrm{R}$, and the equatorial " $\mathrm{H} \mathrm{Ly} \alpha$ bulge" is also observed at a level of roughly $200 \mathrm{R}$.

McConnell et al. [1980] point out that the dark side emission is greater than can be produced by backscattered solar $\mathrm{H}$ Ly $\alpha$ from interplanetary $\mathrm{H}$ atoms, and they conclude that a fraction of all the dark side emission is produced by charged particle excitation. McConnell et al. also present data showing equatorial H Ly $\alpha$ emission at levels of several kilorayleighs extending nearly $20^{\circ}$ in longitude onto the dark hemisphere at the dawn terminator (the dusk terminator was not observed), although they were unable to explain this phenomenon as a result of scattering processes. Thus the electroglow process may occur in the dark atmosphere also, albeit at a reduced level, in contrast with earlier assumptions that it is restricted to the sunlit atmosphere. The equatorial regions between $\pm 30^{\circ}$ latitude and within $20^{\circ}$ of the terminator, observed by McConnell et al., are connected to conjugate magnetic points in the dayside atmosphere at most longitudes due to the significant declination angle of Jupiter's magnetic field [Acuna et al., 1983]. Photoelectrons and ions can freely flow into these shadowed regions from the conjugate illuminated regions with correspondingly high ionospheric ion and electron densities. The observation therefore indicates that electroglow can operate in the absence of sunlight but in the presence of substantial ionospheric densities. Finally, the bulge emission region rotates with the same period as, and is centered on the equator of, the magnetic field rather than the atmosphere (i.e., it is fixed in system III longitude, not systems I or II, [Clarke et al., 1981a; Dessler et al., 1981]. This implies a magnetic dependence of the electroglow mechanism.

The subsolar point integrated brightness of the $\mathrm{H}_{2}$ Lyman band emissions observed with IUE is $1.4 \mathrm{kR}$ [Clarke et al., 1982], and the integrated Lyman and Werner band brightness observed with UVS is roughly $3 \mathrm{kR}$ [Shemansky, 1985]. The $\mathrm{H}_{2}$ band emissions occur at wavelengths where the incoming solar flux is too weak to produce the observed emission levels by scattering, and therefore they are a direct signature of charged particle excitation. The observed spectrum of these band emissions is a sensitive function of the energy of the exciting charged particles, and the equatorial emission spectrum is consistent with excitation by electrons with energies in the range $10-50 \mathrm{eV}$, as opposed to the $10-$ to $100-\mathrm{keV}$ excitation of the polar aurora [Shemansky, 1985]. The measured atomic carbon emission C I $1657 \AA$ is $300 \mathrm{R}$, which is at least $140 \mathrm{R}$ greater than the reflected solar line. A large abundance of $\mathrm{C}$ atoms (and thereby efficient resonant scattering of the solar line) is unlikely, since $\mathrm{C}$ atoms react rapidly with $\mathrm{H}_{2}$ to form simple hydrocarbon radicals and molecules. This suggests particle excitation (probably of $\mathrm{CH}_{4}$ ) near the homopause, i.e., well below the photoelectron production peak [Clarke et al., 1982].

There is considerable spatial structure to the $\mathbf{H}_{2}$ band emissions on all three planets. The $\mathrm{H}_{2}$ bands on Jupiter do not show a comparable enhancement in the $\mathrm{H}$ Ly $\alpha$ bulge region and were not observed from the dark atmosphere with an upper limit of roughly 0.2 times the subsolar brightness [Sandel et al., 1979]. In addition, an increase in $\mathbf{H}_{2}$ band intensity by roughly a factor of 2 from dawn to dusk across the sunlit equator has been reported by Shemansky [1985]. Shemansky also pointed out that this distribution is consistent with emission from an optically thin solar-controlled layer, with the observed dawn to dusk variation resulting from the angle of the observation. No information about the altitude of the FUV emissions was obtained during the Voyager encounters because of high charged particle radiation levels near closest approach.

\section{Saturn}

The subsolar H Ly $\alpha$ brightness has been measured to be 1-2 kR by the IUE (correcting the measurement of Clarke et $a l .[1981 b]$ for the effects of interplanetary hydrogen emission and absorption) and $3 \mathrm{kR}$ by the UVS [Broadfoot et al., 1981]. This emission can also be explained in magnitude by a reasonable (but very high) atmospheric reflectivity to direct solar $\mathbf{H}$ Ly $\alpha$. There has been no claim of detection of a Saturn H Ly $\alpha$ bulge, although we note that there appears to be an enhancement of $10-15 \%$ at $20^{\circ}$ latitude in the $\mathrm{H} \mathrm{Ly} \alpha$ data of Yelle et al. [1986]. The dark side H Ly $\alpha$ brightness at $350 \mathrm{R}$ is, as at Jupiter, inconsistent with resonant scattering of interplanetary background radiation as the sole mechanism and is reported to have a component due to charged particle excitation. The $\mathrm{H}_{2}$ bands appear at a disk-average brightness of roughly 350 $\mathbf{R}$ from the IUE [Clarke et al., 1982] and a subsolar brightness of $1000 \mathrm{R}$ from the UVS [Yelle et al., 1986], with no emission detected from the dark side at an upper limit of 0.015 times the subsolar brightness. The sunlit $\mathrm{H}_{2}$ emissions are consistent with excitation by $10-$ to $50-\mathrm{eV}$ electrons as at Jupiter. The C I $1657-\AA$ emission is $50 \mathrm{R}$, which is close to unit albedo in that line [Clarke et al., 1982]. The emissions also decrease as compared to the sunlit atmosphere in the shadow of Saturn's rings, where the $\mathrm{H}_{2}$ bands decrease by at least $85 \%$ and the $\mathrm{H}$ Ly $\alpha$ by a comparable amount [Broadfoot et al., 1981]. It is significant that a reduced level of emission may still be present, just as bright H Ly $\alpha$ emission was observed from beyond the dawn terminator on Jupiter. Ionospheric charged particles will also be supplied to the ring-shadowed regions along magnetic field lines from conjugate illuminated regions despite the absence of direct solar EUV radiation.

Especially significant is the measurement of the altitude of the emissions in the UVS limb scan data [Yelle et al., 1986], showing peaks for both $\mathrm{H} \mathrm{Ly} \alpha$ and the $\mathrm{H}_{2}$ bands around the homopause level (roughly equivalent to the Earth's $E$ region) with a decrease toward higher altitudes. Both $\mathrm{H} \mathrm{Ly} \alpha$ and the 
$\mathrm{H}_{2}$ bands show strong limb brightening indicative of optically thin emissions. This is not surprising for the $\mathrm{H}_{2}$ bands, since the atmosphere above the emission peak is optically thin to the majority of these emissions, but the vertical optical depth at the center of the $\mathrm{H}$ Ly $\alpha$ line above the observed peak altitude is of the order of $10^{4}$. The atmosphere is optically thin, however, to photons emitted with a Doppler shift of a few times $0.1 \AA$ from the center of the $\mathrm{H}$ Ly $\alpha$ line. The interpretation of Yelle et al. is that the $\mathrm{H} \mathrm{Ly} \alpha$ is produced by fast protons and/or fast $\mathbf{H}$ atoms, which radiate following charge exchange with enough velocity to Doppler-shift their emission from the line center. Only a few kilometers per second motion is required to accomplish this. We agree with the Doppler shift interpretation, although the magnetospheric particle source assumed by Yelle et al. is not essential to the argument. The interpretation of proton-excited $\mathrm{H}$ Ly $\alpha$ contrasts with an earlier interpretation of electron excitation near the exobase, as proposed for Jupiter by Shemansky [1985]. However, no brightening in the exosphere has been observed in the limb scan data on either Saturn or Uranus. Electron impact can dissociate $\mathrm{H}_{2}$, producing fragments in the $2 S$ and $2 P$ states. The kinetic energy of the fragments is roughly 0.1 $\mathrm{eV}$, or $5 \mathrm{~km} / \mathrm{s}$, sufficient to Doppler-shift the $\mathrm{H} \mathrm{Ly} \propto$ out of self-absorption by $\mathrm{H}$ atoms [Lee et al., 1982]. However, the contribution from dissociative excitation of $\mathrm{H}_{2}$ at the electroglow peak altitude on Uranus has been limited to $200 \mathrm{R}$ or less by the observed brightness of the $\mathbf{H}_{2}$ emission [Broadfoot et al., 1986].

There are several reasons to reject the idea of electroglow excitation by precipitating particles, some of which have been discussed by Yelle et al. [1986]. For electrons, energies of 1-100 keV are required to penetrate to the observed peak emission altitude, and these particles would produce an easily identified signature through excitation of $\mathrm{H}_{2}$ band emissions similar to the polar aurora, yet these are not observed [Y $Y$ ung et al., 1982]. In addition, along low-latitude magnetic field lines which are occulted by Saturn's rings there is a measured depletion of trapped charged particles, but a corresponding decrease in low-latitude electroglow brightness is not observed. However, the optically thin $\mathrm{H} \mathrm{Ly} \alpha$ emission suggests that a large fraction of that emission may be proton excited. Finally, we note that there is an observed decrease in all emissions on Saturn from dawn to dusk (opposite to the sense of the observed variation on Jupiter) at a level of $20-30 \%$, but this may also be consistent with a solar-controlled optically thin emitting layer appearing to vary from dawn to dusk due to the geometry of the observation. The intrinsic emission brightness (assuming an optically thin layer) decreases with increasing latitude with roughly a cosine dependence [Yelle et al., 1986].

\section{Uranus}

Although the $\mathrm{H} \mathrm{Ly} \propto$ brightness of Uranus is comparable to that of Saturn, it is once again difficult to separate the contributions from scattering processes and particle excitation. The sunlit disk average $\mathrm{H} \mathrm{Ly} \alpha$ brightness has averaged $1.4 \mathrm{kR}$ over 4 years of IUE observations (which includes the auroral emission [Clarke et al., 1986]) and was measured at $1.5 \mathrm{kR}$ by the UVS during the Voyager encounter (not including auroral emission [Broadfoot et al., 1986]). The dark hemisphere $\mathrm{H} \mathrm{Ly}$ $\alpha$ brightness is $170 \mathrm{R}$, again suggesting some charged particle excitation. The subsolar $\mathrm{H}_{2}$ brightness is 400-1800 R depending on the assumed contribution of the $\mathrm{H}_{2} a-b$ continuum, and no detection of diffuse $\mathrm{H}_{2}$ emission from the dark side was reported, although without a specified upper limit [Broadfoot
TABLE 1a. Solar EUV $(E>15 \mathrm{eV})$ Energy Input at Times of Voyager 2 Encounters

\begin{tabular}{lcccc}
\hline Planet & Day/Year & $\begin{array}{c}\text { Solar EUV Flux, } \\
\text { erg cm }{ }^{-2} \mathrm{~s}^{-1}\end{array}$ & $\begin{array}{c}\text { Emission Flux } \\
\operatorname{erg~cm}^{-2} \mathrm{~s}^{-1}\end{array}$ & $\begin{array}{c}\text { Emission/ } \\
\text { Solar }^{c}\end{array}$ \\
\hline Jupiter & $190 / 1979$ & 0.14 & $0.07-0.13$ & $3-6$ \\
Saturn & $238 / 1981$ & 0.056 & $0.022-0.048$ & $3-6$ \\
Uranus & $024 / 1986$ & 0.0036 & $0.014-0.045$ & $25-80$ \\
\hline
\end{tabular}

${ }^{a}$ Solar EUV fluxes at each planet are based on data of Hinteregger et al. [1981] scaled to day of Voyager encounter by solar $10.7-\mathrm{cm}$ flux (W. Hoegy, personal communication, 1987).

${ }^{b}$ The estimates of particle-excited emission assume the values given in Table $1 b$.

${ }^{c}$ Observed electroglow emission flux divided by photoelectron excitation (assumed to be solar EUV flux times 15\%).

et al., 1986]. The altitude distribution of the emissions is similar to that for Saturn, with the peak for both $\mathrm{H}$ Ly $\alpha$ and $\mathrm{H}_{2}$ bands just above the homopause and decreasing with altitude up to the exosphere. It has been reported that the excitation energy at Uranus is lower than at the other two planets (a Maxwellian distribution with a peak at $3 \mathrm{eV}$ [Broadfoot et al., 1986]). However, more recent work suggests that the reported $\mathrm{H}_{2} a-b$ continuum may alternately be attributed to Rayleighscattered sunlight, implying that the $\mathrm{H}_{2}$ excitation energy is actually closer to the 10 - to $50-\mathrm{eV}$ range observed at Jupiter and Saturn [Yelle et al., 1987].

Most important, the emitted FUV energy is considerably greater than the energy available for UV emission from direct photoelectron excitation, assuming that $15 \%$ of the total solar EUV energy $(E>15 \mathrm{eV})$ goes into photoelectron excitation of FUV emission [Cravens et al., 1975]. This level of energy deficit is characteristic of all three outer planets (see Table 1a); i.e., the input photoelectron energy must be amplified to produce the observed emission. It is interesting that the amplification factor listed in the last column of Table $1 a$ is comparable at Jupiter and Saturn but nearly an order of magnitude larger at Uranus. This conclusion is subject to the large uncertainties in separating the electroglow portion of both $\mathbf{H}$ Ly $\alpha$ and $\mathbf{H}_{2}$ emissions at Uranus discussed above, however. Direct excitation by photoelectrons can be an important contributor and closely mimics the observed properties on all three planets [Prange, 1986], but there must be an additional energizing mechanism. This is the enigma that led to the recognition of a fundamentally new process, and the new name "electroglow."

\section{Elements of the Reguired Emission Process}

In view of the preceding observations, the required characteristics of the emission process are (1) the existence of a sunlight "trigger" plus an additional energy source, (2) a diffuse low- and mid-latitude process, which may include weak $\mathrm{H}$ Ly $\alpha$ emission from the dark hemisphere, (3) charged particle excitation, with an energy of $10-50 \mathrm{eV}$, by both electrons (for $\mathrm{H}_{2}$ band emissions) and protons (for optically thin $\mathrm{H} \mathrm{Ly} \alpha$ emis-

TABLE $1 b . \quad$ Assumed Brightness Values

\begin{tabular}{lccr}
\hline & $\mathrm{H} \mathrm{Ly} \alpha$ & $\mathrm{H}_{2}$ & \multicolumn{1}{c}{ Total } \\
\hline Jupiter & $3-5$ & $1.4-3.0$ & $4.4-8.0$ \\
Saturn & $1-2$ & $0.35-1.0$ & $1.35-3.0$ \\
Uranus & $0.5-1.0$ & $0.40-1.8$ & $0.9-2.8$ \\
\hline
\end{tabular}

Units are kilorayleighs. 
sion), (4) peak excitation at or just above the homopause, at neutral densities of $10^{12}$ to $10^{13} \mathrm{~cm}^{-3}$, where the mean free path for a low-energy electron is of the order of $1 \mathrm{~m}$, and (5) some sort of magnetic control, if the magnetic-field-related $\mathbf{H}$ Ly $\alpha$ bulge on Jupiter is interpreted as electroglow.

Since the peak in emission is observed near the altitude of maximum photoelectron production, the emission region covers the sunlit hemisphere, and decreases rapidly in the absence of sunlight, it is natural to interpret the production of photoelectrons (and ions) as providing the sunlight trigger. Given the preceding constraints on precipitating particles, the particle acceleration processes must occur in situ and accelerate both electrons and ions, i.e., electric field acceleration in a region of neutral density and magnetic field strength comparable to the Earth's $E$ and $F$ regions. This interpretation is very different from previous ones involving precipitating particles, in the sense that low-energy particles are accelerated in the presence of collisions with neutrals, rather than highenergy particles being slowed down by collisions.

Strong electric currents in the $E$ and $F$ regions of the Earth's ionosphere are produced by an atmospheric dynamo, whose driving force stems from charge separation by the interaction of neutral winds with ionospheric plasma. A derivation and review of dynamo theory and its manifestations is given by Rishbeth and Garriott [1969]. If $\boldsymbol{\Omega}_{e}$ is the electron gyrofrequency and $v_{e n}$ is the collision frequency of electrons with neutrals,

$$
\Omega_{e}=e B / m_{e} \quad v_{e n}=v_{e n} \sigma_{e n}
$$

then the ionosphere divides into two regions. At high altitudes (low densities) $\Omega_{e} \gg v_{e n}$, and individual electron motions are confined to magnetic field lines. At low altitudes (high densities) $\nu_{e n} \gg \Omega_{e}$, and the electrons are dragged across magnetic field lines with the wind motion of the neutral atmosphere. Similar quantities $\Omega_{i}$ and $v_{t n}$ can be defined for ions, except

$$
\Omega_{i}=\left(m_{e} / m_{i}\right) \Omega_{e} \quad v_{1 n}=\frac{\sigma_{i}}{\sigma_{e}}\left(\frac{m_{e}}{2 m_{t}}\right)^{1 / 2} v_{e n}
$$

for ions and electrons of equal energy (i.e., assuming that $T_{i}=$ $T_{e}$ ). The ratio of collision frequency to gyrofrequency is therefore higher for ions than for electrons by the ratio

$$
\frac{\sigma_{1}}{\sigma_{e}}\left(\frac{m_{i}}{2 m_{e}}\right)^{1 / 2}
$$

and neutral collisions dominate particle motions at higher altitudes for ions than for electrons. In the intermediate alti-

TABLE 2a. Ionospheric Dynamo Conditions on the Planets

\begin{tabular}{lllc}
\hline & Homopause Density & $v_{e n} / \Omega_{e}$ & $v_{i n} / \Omega_{i}$ \\
\hline Jupiter $^{a}$ & $n_{\mathrm{H}_{2}}=5 \times 10^{13} \mathrm{~cm}^{-3}$ & 0.0056 & 3.4 \\
Saturn $^{b}$ & $n_{\mathrm{H}_{2}}=1 \times 10^{12}$ & 0.0025 & 1.1 \\
Uranus $^{c}$ & $n_{\mathrm{H}_{2}}=5 \times 10^{13}$ & 0.12 & 45 \\
Earth $^{d}(90 \mathrm{~km})$ & $n_{\mathrm{H}_{2}}=4 \times 10^{13}$ & 0.035 & 340 \\
\hline
\end{tabular}

Here, $v_{e n}$ is the electron-neutral collision frequency, and $\Omega_{e}$ is the electron gyrofrequency.

${ }^{a}$ Assuming atmospheric model of Festou et al. [1981], where $T_{\text {homopause }}=200 \mathrm{~K}$.

${ }^{b}$ Assuming atmospheric model of Smith et al. [1983], $T_{h}=400 \mathrm{~K}$.

'Assuming atmospheric model of Broadfoot et al. [1986], $T_{h}=500$ $\mathrm{K}$.

${ }^{d}$ Assuming $T(D$ region $)=200 \mathrm{~K}$.
TABLE $2 b$. Earth Ionospheric Conditions

\begin{tabular}{cccc}
\hline $\begin{array}{c}\text { Altitude, } \\
\mathrm{km}\end{array}$ & $\begin{array}{c}\text { Neutral } \\
\text { Density, } \\
\mathrm{cm}^{-3}\end{array}$ & $\begin{array}{c}\text { Electron } \\
\text { Density, } \\
\mathrm{cm}^{-3}\end{array}$ & Region \\
\hline 90 & $4 \times 10^{13}$ & $2 \times 10^{4}$ & $D$ \\
110 & $1 \times 10^{12}$ & $\leq 1 \times 10^{5}$ & $E$ \\
200 & $5 \times 10^{9}$ & $\leq 2 \times 10^{5}$ & $F_{1}$ \\
300 & $1 \times 10^{8}$ & $\leq 10^{6}$ & $F_{2}$ \\
\hline
\end{tabular}

tude range where

$$
v_{e n} / \Omega_{e} \ll 1 \quad v_{i n} / \Omega_{i} \simeq 1
$$

the ions will be dragged along by a neutral wind while the electrons remain fixed on magnetic field lines, resulting in charge separation in the direction of the neutral wind at dawn/dusk boundaries where the winds reverse. The numerical values for $v_{e n} / \Omega_{e}$ and $v_{t n} / \Omega_{t}$ at the homopause on the three outer planets are listed in Table $2 a$ (assuming the ions to be protons) and compared with corresponding properties of the Earth's ionosphere (Table $2 b$ ). Collision frequencies are calculated using the relations given by Banks and Kockarts [1973] and electron scattering cross sections from Trajmar et al., [1983], and Earth $D$ region ion frequencies are calculated for $\mathrm{O}_{2}{ }^{+}$on $\mathrm{N}_{2}$.

On the Earth the peak dynamo currents are measured at altitudes near $110 \mathrm{~km}$ (somewhat higher than the altitude of maximum charge separation [cf. Rishbeth and Garriott, 1969]) and extend to much higher altitudes [Pfaff, 1986]. From Table 2 the equivalent conditions on Uranus, Saturn, and Jupiter occur just above the homopause. We know that dynamos will operate in the outer planet atmospheres, subject to the neutral wind velocity, the strength of the magnetic field, and the charged particle density. The question is how much of the energy will go into FUV radiation.

Acceleration of the magnetized electrons requires fieldaligned potentials. Collisional drag of protons at $100 \mathrm{~m} / \mathrm{s}$ gives only $5 \times 10^{-5} \mathrm{eV}$, which is not sufficient for excitation of UV emissions and suggests that the ions are also accelerated by field-aligned potentials. Field-aligned (Birkeland) currents in the Earth's $F$ and $E$ regions result from current closure along the highly conductive magnetic field lines driven by $E$ and $F$ region polarization due to the dynamo action [Rishbeth, 1971]. The basic ionospheric circuit is shown in Figure 1 for mid-latitude and equatorial cases. The conductivity for electron flow along field lines is extremely high, and the Pederson $\left(j_{E}\right)$ conductivity in the $E$ region is also high and carried mainly by protons. Ion Pedersen conductivity provides crossfield line current closure in both the $E$ and $F$ region legs of the circuit. The $E$ region conductivity is greatly reduced at night by recombination, so dynamo-driven Birkeland currents are larger during the day relative to a fixed neutral wind velocity [Rishbeth, 1971]. Sufficiently high Birkeland currents can give rise to plasma instabilities which inhibit the bulk of the electron drift at the expense of accelerating high-energy electrons to superthermal energies, a phenomenon known as anomalous resistivity. Anomalous resistivity has long been thought to contribute to the acceleration of auroral particles on Earth up to tens of $\mathrm{keV}$ [Swift, 1965]. We propose that a weaker version of this phenomenon at low and middle latitudes accelerates photoelectrons (and ions) above some critical energy along hundreds of kilometers parallel to $B$ in the ionosphere to produce the electroglow emissions. The exciting charged 


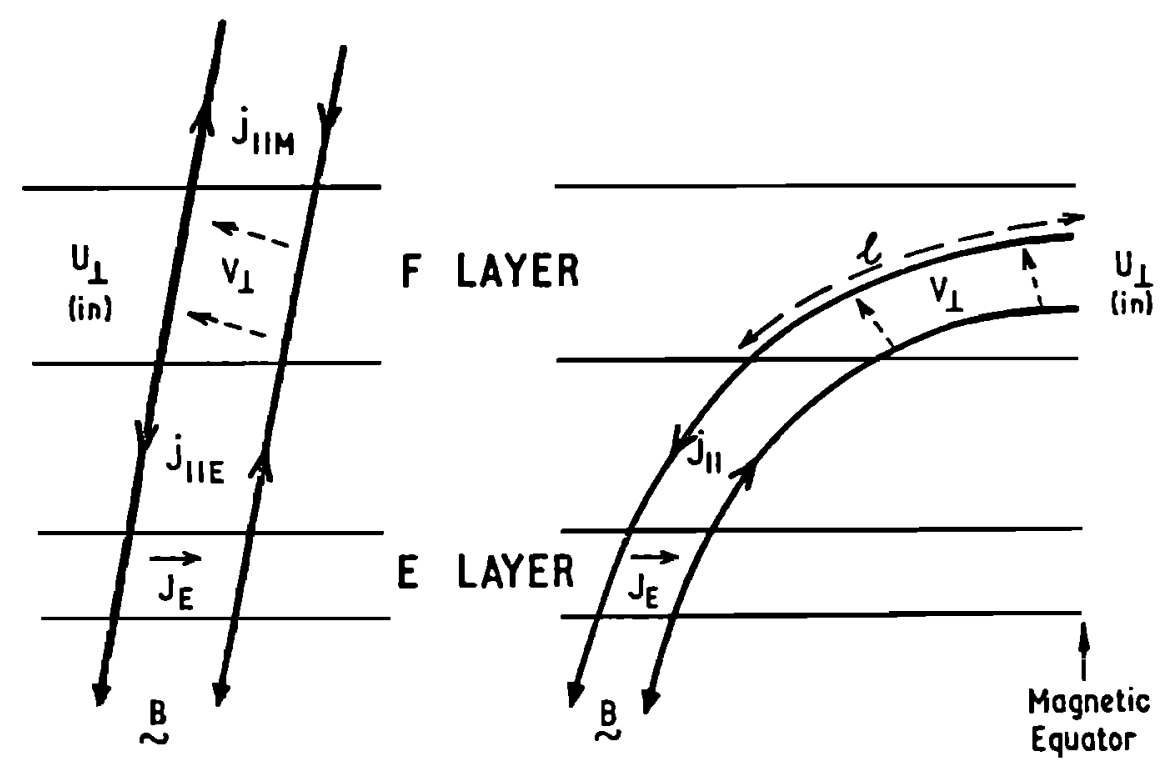

Fig. 1. Schematic drawing of the ionospheric circuits $(a)$ at mid-latitudes and $(b)$ at the equator (taken from Rishbeth [1971]), showing the currents driven by $F$ region neutral winds $U$ directed into the plane of the figure. Rishbeth's caption states "(a) Midlatitude case. The field-aligned current comprises a current $j_{\| E}$ linking with the $E$-layer current and a magnetospheric current $j_{\| M}$. (b) Equatorial case. No current flow across the magnetic equator (right-hand edge of diagram) if there is symmetry about the equator. The length $l$ of a field line within the $F$-layer is greater than in the mid-latitude case."

particles therefore represent a nonlocal thermodynamic equilibrium (LTE) population in the ionosphere, since anomalous resistivity preferentially accelerates particles in the high-energy portion of the distribution.

We emphasize that it is the presence of Birkeland currents in the ionosphere, rather than direct dynamo action, that leads to FUV emissions by the mechanism of anomalous resistivity. Any contributions to Birkeland currents from magnetospheric processes or from magnetic conjugate regions with relatively higher ionospheric densities will lead to locally enhanced FUV emission. It is the observation of electroglow throughout the sunlit atmospheres that indicates substantial excitation by the more global dynamo processes. The detailed calculation of the generation of field-aligned potentials by neutral winds is discussed in a paper by M. K. Hudson et al. (A Dynamo theory of Electroglow Emissions from the Outer Planets, submitted to Journal of Geophysical Research, 1987), hereinafter referred to as H87. They show that for the case of Uranus, assuming a wind velocity of twice the maximum observed cloud velocity with respect to the magnetic field $(400 \mathrm{~m} / \mathrm{s}$ [Smith et al., 1987]), total energies in excess of collisional losses of $10-20 \mathrm{eV}$ may be gained by photoelectrons and ions over reasonable path lengths in the ionosphere. In the next sections we explore the consequences of accelerating low-energy electrons and protons and of dynamo-produced field-aligned potentials in an $\mathrm{H}_{2}$ atmosphere.

\section{The Linear Acceleration of Low-Energy ELECTRONS AND PROTONS}

Photoelectrons are produced in the outer planet atmospheres by absorption of solar ionizing radiation:

$$
\begin{aligned}
\mathrm{H}_{2}+h v & \rightarrow \mathrm{H}_{2}^{+}+e \\
& \rightarrow \mathrm{H}^{+}+\mathrm{H}+e \\
\mathrm{H}+h v & \rightarrow \mathrm{H}^{+}+e \\
\mathrm{He}+h v & \rightarrow \mathrm{He}^{+}+e
\end{aligned}
$$

For simplicity we shall ignore the photoionization of $\mathrm{CH}_{4}$ and other hydrocarbons. The fate of photoelectrons in a pure hydrogen atmosphere has been investigated by Cravens et al. [1975]. Photoelectrons lose energy in collisons with $\mathbf{H}_{2}$ by electronic and vibrational excitation, ionization, and dissociation and are eventually thermalized. Figure 2 shows the overall loss function $L(E)$ for electrons in $\mathrm{H}_{2}$ and the contributions to $L(E)$ from the various processes. The effect of elastic collisions is not included in computing $L(E)$; we will address this issue later. Given the initial energy of an electron, the subsequent energy cascade [Dalgarno et al., 1969] can be described by the equation

$$
d E / d x=\alpha-n L(E)
$$

where $d E / d x$ is the rate of energy loss per unit length $(\mathrm{eV} / \mathrm{cm})$, $n$ is the number density of ambient gas (molecules $\mathrm{cm}^{-3}$ ), and $L(E)$ is the energy loss function $\left(\mathrm{cm}^{2} \mathrm{eV}\right)$. An electric force $\alpha$ $(\mathrm{eV} / \mathrm{cm})$ may also be acting on the photoelectrons. Since $L(E)$ and $n$ are always positive, the second term describes a monotonic downward cascade of energy. However, assuming that $\alpha>0$, there may be an increasing electron energy if successive paths are coaligned (i.e., the electron scattering is forward 


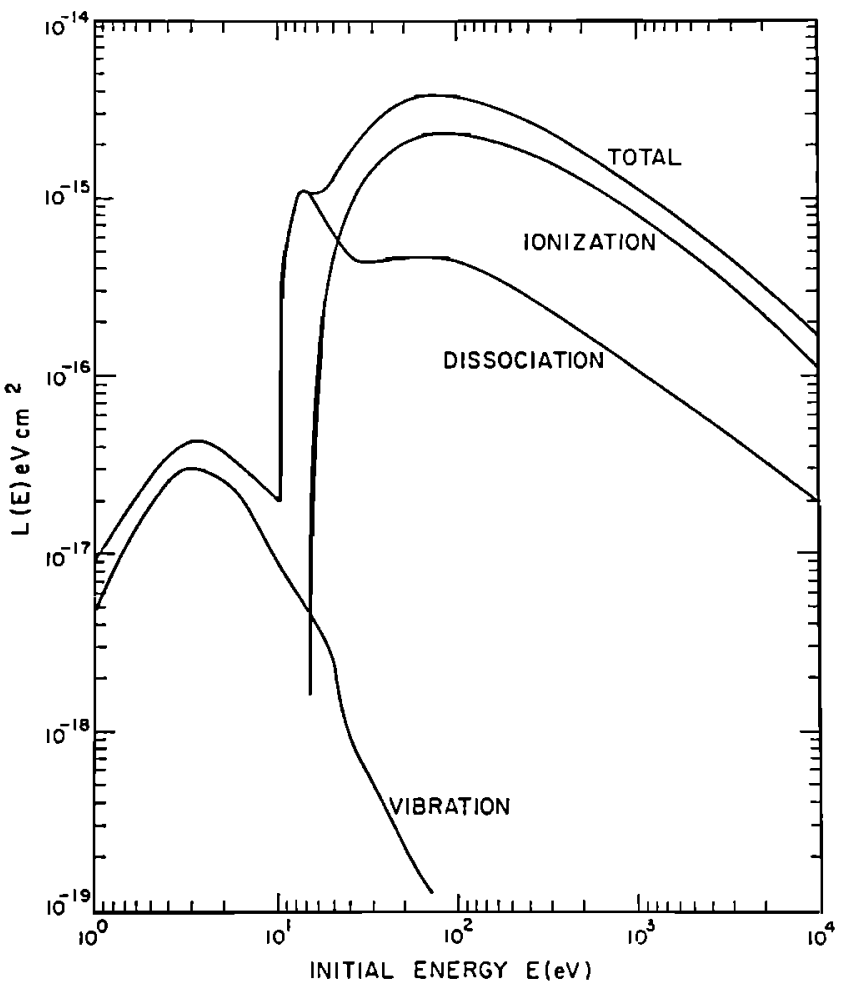

Fig. 2a. The electron energy loss function $L(E)$ calculated by $\mathrm{Cra}$ vens et al. [1975] for electrons in $\mathrm{H}_{2}$ (see text).

peaked). The mean electron energy increases if

$$
\alpha>n L(E)
$$

Table 3 gives numerical values of the critical force $\alpha_{c}=n L(E)$ for electron energies and neutral densities typical in the regions of maximum electroglow emission. Referring to Table 3, at $n=10^{12} \mathrm{~cm}^{-3}$ and electron energy up to $E=10 \mathrm{eV}$, a local electric field of $2 \mathrm{mV} / \mathrm{m}$ is sufficient to compensate for the collisional loss processes so that the electron energy will be maintained.
Inspection of Figure 4 of Broadfoot et al. [1986] shows that the electroglow emissions decay rapidly across the dark limb, indicating a photoelectron time constant of the order of an hour or less. From Figure $2 b$ we note that the cross section for elastic scattering is much larger than that for inelastic scattering. However, there is the complication that elastic scattering can be highly anisotropic and tends to be forward peaked at larger energies. A convenient measure of the anisotropy of elastic scattering is the asymmetry parameter

$$
g=\frac{\int_{-1}^{1} \sigma(\mu) \mu d \mu}{\int_{-1}^{1} \sigma(\mu) d \mu}
$$

where $\sigma(\mu)$ is the differential cross section and $\mu=\cos \theta(\theta$ is the scattering angle). The asymmetry $g$ is a strong function of energy. Using the data from Table 4 of Trajmar et al. [1983], we compute

$$
\begin{array}{ll}
g=0 & E \leq 3 \mathrm{eV} \\
g \geq 0.8 & E \geq 10 \mathrm{eV}
\end{array}
$$

where the range of $g$ is -1 to 1 , with 0 representing no asymmetry and 1 complete forward scattering. For low-energy (a few electron volts) electrons, elastic scattering is isotropic, while at higher energies the scattering is highly anisotropic. Thus for an electron at low energy, elastic scattering is nearly an isotropic random walk, and electrons do not gain energy from any electric field. The high cross section for vibrational excitation of $\mathrm{H}_{2}$ near $3 \mathrm{eV}$ (see Figure $2 b$ ) will act as an additional sink for the electron energy. At higher energy where elastic scattering is forward peaked, the gas is effectively transparent, and the effects of elastic collisions can be ignored. This combination of circumstances will also lead to the relaxation of high-energy photoelectrons. A random walk in energy space may place the energy of the photoelectron sufficiently low that it is lost from the acceleration process. It therefore appears that the production of high-energy photoelectrons is necessary

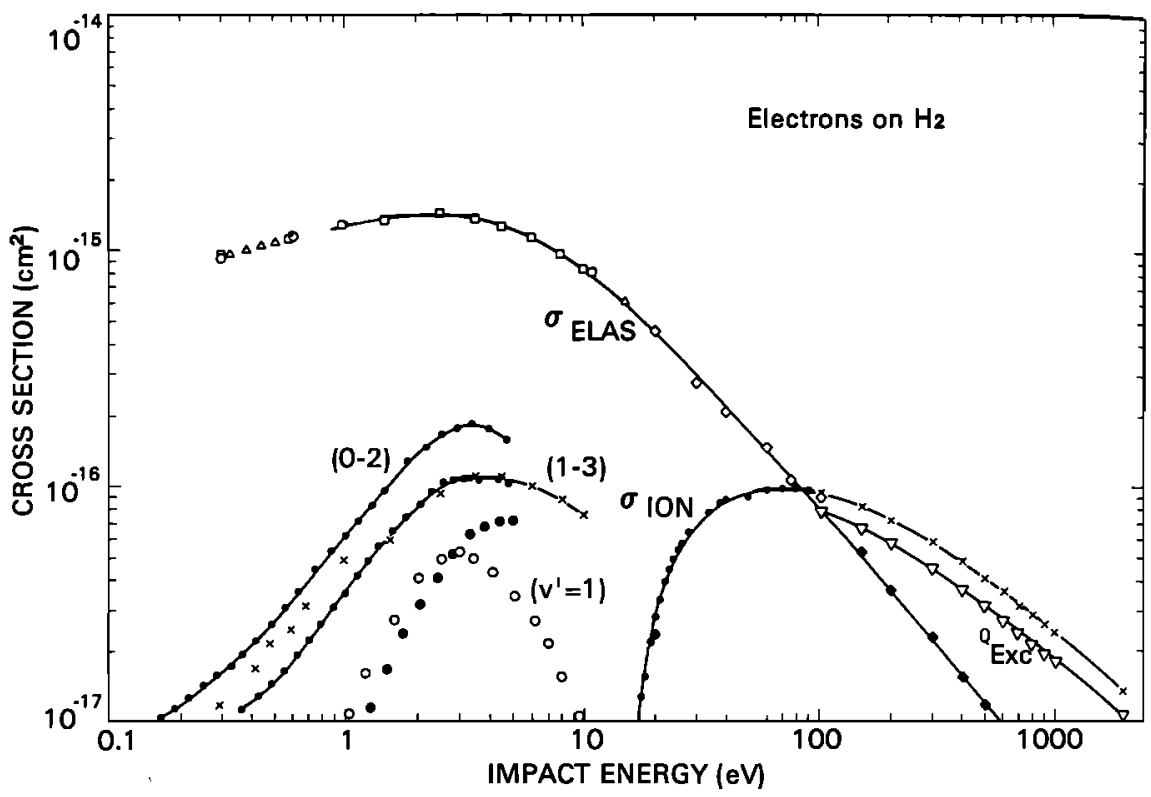

Fig. 2b. Various cross sections for electron collisions with $\mathrm{H}_{2}$ molecules, adapted from Trajmar et al. [1983]. The curves marked (0-2), (1-3), and $\mathrm{v}^{\prime}=1$ are for vibrational excitation, $\sigma_{\mathrm{ELAS}}$ is for elastic scattering, and $\sigma_{\mathrm{ION}}$ is for ionization of $\mathrm{H}_{2}$. The cross section for electronic excitation, $\sigma_{\mathrm{Exc}}$ follows $\sigma_{\text {ION }}$ to within a factor of 2 . 
TABLE 3. Numerical Values of the Critical Force $\alpha_{c}$

\begin{tabular}{rccc}
\hline $\begin{array}{c}E, \\
\mathrm{eV}\end{array}$ & $\begin{array}{c}n=10^{11} \\
\mathrm{~cm}^{-3}\end{array}$ & $\begin{array}{c}n=10^{12} \\
\mathrm{~cm}^{-3}\end{array}$ & $\begin{array}{c}n=10^{13} \\
\mathrm{~cm}^{-3}\end{array}$ \\
\hline 1 & 0.1 & 1.0 & 10 \\
3 & 0.5 & 5.0 & 50 \\
10 & 0.2 & 2.0 & 20 \\
20 & 10 & 100 & 1000 \\
\hline
\end{tabular}

Values are in millielectron volts per meter.

to permit any acceleration process to function and that the particle acceleration will rapidly decrease in the absence of EUV sunlight.

It is also clear from Figure $2 b$ that electrons accelerated in the range 1-6 eV will preferentially lose their energy in the excitation of vibrational transitions of $\mathrm{H}_{2}$, which will contribute to heating the local neutral atmosphere. Since roughly one in five collisions results in vibrational excitation at $3 \mathrm{eV}$ and elastic scattering is largely isotropic at the lower energies, particles below roughly $5 \mathrm{eV}$ contribute to heating with a high efficiency. The ratio of heating to UV excitation in the electroglow process then depends on the lower cutoff energy above which anomalous resistivity will accelerate electrons (i.e., the lower the cutoff, the more heating compared to UV radiation). Linear acceleration of the entire photoelectron distribution would result in too much heat compared to UV radiation to satisfy the observations, and this has been a contributing factor in leading us to propose a non-LTE process for the electroglow excitation. Estimates of very high temperature thermal distributions of electrons also indicate too much heating compared to radiation (J. H. Waite, personal communication, 1987). The vibrational excitation is potentially capable of heating the upper atmospheres of all three planets to the observed temperatures of $400-1200 \mathrm{~K}$, although the magnitude of this contribution compared to other heating processes (such as inertial gravity waves) has yet to be established.

The fate of those electrons that are accelerated can be seen in Figure 2. At an energy of roughly $10-15 \mathrm{eV}$ a steep increase in $L(E)$ is encountered, representing cross-section increases for dissociation, ionization, and excitation of the Lyman and Werner band systems of $\mathrm{H}_{2}$. Near $10 \mathrm{eV}$ the threshold for $\mathbf{H}_{2}$ dissociation is reached, and just above $11 \mathrm{eV}$ is the threshold for resonant excitation of excited state $\mathrm{H}_{2}{ }^{-}$leading to Lyman band emission (J. Ajello, personal communication, 1987). This process, in particular, produces vibrational features observed in the UVS spectra of Uranus that are not produced by direct excitation of the $B$ state. In addition, the cross section for elastic scattering is decreasing toward higher energies and will be exceeded by the combined (ionization plus excitation) cross section at roughly $40-50 \mathrm{eV}$. At this energy an electron will be more likely to directly excite or ionize an $\mathbf{H}_{2}$ molecule with each collision, and it thus represents a practical upper limit to the energy an electron will achieve before losing $10-15 \mathrm{eV}$ to one of these processes. This is the $\mathrm{H}_{2}$ equivalent of the classic Franck-Hertz experiment, which demonstrated the existence of quantum levels in atoms through a decrease in current and the initiation of $2537 \AA$ radiation from $\mathrm{Hg}$ gas at the $4.9-\mathrm{eV}$ potential of the first excited state [Franck and Hertz, 1965]. This phenomenon does not occur with the same magnitude in the Earth's atmosphere, because the elastic scattering cross sections for $\mathrm{N}_{2}$ and $\mathrm{O}_{2}$ remain several times greater than those for excitation at all energies. In an $\mathrm{H}_{2}$ atmosphere, relatively more of the energy from linearly accelerated electrons is dissipated as radiation and relatively less as Joule heating, and the $\mathrm{H}_{2}$ electroglow will naturally exhibit a spectrum characterized by 10 - to $50-\mathrm{eV}$ excitation if it is excited by electrons accelerated slowly $(\nabla E / E \ll 1 / x$, where $x$ is the mean free path) from lower energies. A Monte Carlo program is being developed to model the processes described here in detail.

It is more difficult to estimate the excitation of $\mathrm{H} \mathrm{Ly} \alpha$ emission by accelerated protons than to estimate electron excitation of $\mathbf{H}_{2}$ band emissions, mainly because of a lack of laboratory cross-section data on the interaction of protons with $\mathrm{H}_{2}$ molecules at low energies $(E<50 \mathrm{eV})$. At a neutral density of $10^{12} \mathrm{~cm}^{-3}$ and charge exchange cross section of $10^{-17} \mathrm{~cm}^{2}$ (extrapolating the data of Cramer [1961]), the proton mean free path with respect to charge exchange is 1 $\mathrm{km}$, as opposed to a mean free path with respect to elastic scattering of roughly $10 \mathrm{~m}$. Given an electric field of 1-10 $\mathrm{mV} / \mathrm{m}$, the energy gained by the proton before charge exchanging will be $1-10 \mathrm{eV}$, and there will be a very significant population of these fast $\mathrm{H}$ atoms. Since a Doppler shift of a few electron volts is sufficient to produce optically thin $\mathrm{H} \mathrm{Ly} \alpha$ emission, electron excitation of these fast $\mathbf{H}$ atoms will contribute to the observed optically thin emission as well as $\mathrm{H}$ atoms which are formed by charge exchange into the first excited state. In either event, the Doppler shift will be in the direction of the proton acceleration. These fast $\mathbf{H}$ atoms will also resonantly scatter incident $\mathrm{H}$ Ly $\alpha$ photons in the wings of the solar line, thereby enhancing the atmospheric albedo to solar H Ly $\alpha$ and broadening the planetary emission line. The contribution of these fast $\mathrm{H}$ atoms will be limited by their loss of energy in collisions with $\mathrm{H}_{2}$ molecules in a distance of 10-100 m. Finally, Doppler-shifted H Ly $\alpha$ may be produced by electron impact dissociation of $\mathrm{H}_{2}$ in cases where the electron has sufficient energy to impart a few electron volts in kinetic energy to the resulting $\mathrm{H}$ atom(s), in addition to electronic excitation of the resulting $\mathrm{H}$ atom(s).

\section{CONSEQUENCES OF THE DYNAMO THEORY}

In this section we will discuss the expected FUV radiation distribution which can arise from dynamo-produced electric fields on the outer planets largely by analogy with what has previously been observed on the Earth. Although the applicability of such an analysis has limits, many of the general characteristics of electroglow are directly analogous to phenomena observed on the Earth.

The brightness of dynamo-produced electroglow emission will depend on the photoelectron and ion densities and energy spectra, on the direction and magnitude of the neutral wind, on the dip angle of the magnetic field, and on the contribution to Birkeland currents from magnetospheric convection. The brightness distribution will also depend on global circulation patterns of both the neutral atmosphere and the ionospheric plasma. The general characteristics expected for purely dynamo-driven emission (as discussed also by H87) are a decreasing level of excitation toward higher latitudes, possible dawn-dusk asymmetries depending on dawn-dusk variation in neutral wind speed, and the existence of an equatorial electrojet as observed on the Earth. Contributions to the excitation process from magnetospheric convection would reflect the plasma conditions along the path of the magnetic field lines through the magnetosphere, which would vary from planet to planet, although low- and mid-latitude effects would 
generally be attributable to ionospheric and ring-associated plasmas, respectively.

One problem with a simple dynamo explanation for electroglow is the observation of equatorial brightenings in the $\mathrm{H} \mathrm{Ly}$ $\alpha$ emission but not in the $\mathbf{H}_{2}$ emission. Electric fields will, of course, accelerate both electrons and protons (and excite both $\mathrm{H}_{2}$ bands and $\mathrm{H}$ Ly $\alpha$ ), and by direct analogy to the Earth's equatorial electrojet, a significant equatorial enhancement in Birkeland currents and potentials would be expected. As indicated in Figure $1 b$, and discussed further by $\mathrm{H} 87$, Birkeland currents are upward below and downward above the equatorial $F$ peak. The downward current region is more extended in altitude at the equator and correspondingly more extended in latitude away from the equator. Protons will be accelerated downward in the downward current region, and electrons will be accelerated downward in the upward current region toward increasing neutral density and relatively increasing electroglow emission. The proton-generated H Ly $\alpha$ emissions associated with the equatorial dynamo may thus be more extensive, in latitude and altitude, and more intense when integrated over latitude, than the electron-produced $\mathrm{H}_{2}$ emissions. However, the latter conclusion is moderated by the fact that the potential drop is greater in the region of downward electron acceleration (H87).

As an alternative mechanism, the Earth's dynamo also leads to an equatorial fountain [Hanson and Moffett, 1966] and tropical arc emission by recombination of ions, which can explain $\mathbf{H}$ Ly $\alpha$ production by proton recombination without $\mathrm{H}_{2}$ emission by electron excitation. There are several lines of evidence for an equatorial anomaly on Jupiter at the location of the observed $\mathrm{H} \mathrm{Ly} \alpha$ bulge from Pioneer and Voyager radio occultation observations [Mahajan, 1981], and there has been preliminary modeling of a solar-driven dynamo [Tan, 1986]. H Ly $\alpha$ emission by recombination would be accompanied by Ly $\beta$ emission at $1025.72 \AA$, and a strong feature appears at this wavelength in the UVS spectra of the bulge region. However, this line can be absorbed by (and confused with emission from) a coincident $\mathrm{H}_{2}$ Lyman band at $1025.94 \AA$ [see Shemansky, 1985]. The presently unknown altitude and Doppler profile of the recombination emission line would be necessary to estimate the magnitude of this absorption. Since the magnetic polarities of Jupiter and Saturn are opposite to the polarity of the Earth, the $E$ and $F$ region winds must also be reversed from the Earth for an upward fountain $(\mathbf{v} \times \mathbf{B})$ to exist. In fact, observations of electron density peaks north and south of the equator [Mahajan, 1981] indicate the presence of an upward fountain [Hanson and Moffett, 1966]. We note finally that there is a marginal indication of a double peak in the $\mathrm{H}$ Ly $\alpha$ emission from Jupiter's bulge region in the data of Dessler et al. [1981], suggesting that the H Ly $\alpha$ emission has a spatial distribution similar to the Earth's tropical arc emissions.

Thermospheric winds are driven in part by near-UV solar heating of the lower atmosphere and in part by in situ FUV absorption. Since the near-UV solar flux is relatively constant (both short term and long term), this component will not vary greatly with time. Auroral heating will also contribute to thermospheric winds at high latitudes and may dominate the energetics over much of the atmosphere, at least on Jupiter. The auroral component will, of course, be much more variable than the solar component. The sum of these components will determine the global neutral wind patterns, and their estimation will be an important step in evaluating the potential for the dynamo mechanism to produce the electroglow. In view of the large sizes, rapid rotation rates, intense auroral emissions, and decreased solar input at the outer planets, their thermospheric wind patterns should be very different from those on the Earth. Unfortunately, there are presently no observational data on the thermospheric winds from any outer planet. The peculiar magnetic geometries, especially on Uranus, will also complicate the generation of dynamo electric fields. In particular, the extended regions on Jupiter and Uranus of nearly horizontal magnetic field are conducive to the generation of extended regions of tropical arc H Ly $\alpha$ emission, as discussed above. The position of Jupiter's H Ly $\alpha$ bulge region is consistent with the most extended region of horizontal magnetic field along Jupiter's equator [Acuna et al., 1983], and preliminary modeling of the surface field of Uranus indicates an even more extended region of nearly horizontal magnetic field on the presently illuminated hemisphere [Connerney et al., this issue].

Fortunately, several tests of the proposed mechanism should be possible with existing or upcoming instruments. Much can be learned from measurements of the Doppler broadening of the $\mathrm{H} \mathrm{Ly} \alpha$ line which will result from charge exchange by fast protons and fast $\mathbf{H}$ atom excitation. Since a proton energy of only a lew electron volts is required to Doppler-shift the emission to an optically thin distance from the center of the atmospheric line, a spectral resolution of the order of $0.1 \AA$ will be necessary to resolve these motions. This technique can verify the excitation mechanism and distinguish fast proton and $\mathbf{H}$ atom energies parallel and perpendicular to field lines. Limb scan observations on Jupiter to measure the altitude of the emissions can discriminate between exobase excitation and an ionospheric phenomenon similar to Saturn and Uranus. Finally, future planetary encounter missions may include instrumentation to measure the thermospheric winds and/or ionospheric electric fields.

Acknowledgments. We wish to acknowledge helpful discussions with A. Dessler, W.-H. Ip, H. Mayr, J. Moses, D. Shemansky, H. Waite, J. Warren, and R. Yelle. M.K.H. acknowledges support from NASA grant NAGW-809 to Dartmouth College, and Y.L.Y. acknowledges support from NASA grant NSG 7376 to the California Institute of Technology.

The Editor thanks R. V. Yelle and another referee for their assistance in evaluating this paper.

\section{REFERENCES}

Acuna, M. H., K. W. Behannon, and J. E. P. Connerney, Jupiter's magnetic field and magnetosphere, in Physics of the Jovian Magnetosphere, edited by A. J. Dessler, p. 1, Cambridge University Press, New York, 1983.

Banks, P. M., and G. Kockarts, Aeronomy, Academic, Orlando, Fla., 1973.

Broadfoot, A. L., et al., Extreme ultraviolet observations from Voyager 1 encounter with Jupiter, Science, 204, 979, 1979.

Broadfoot, A. L., et al., Extreme ultraviolet observations from Voyager 1 encounter with Saturn, Science, 212, 206, 1981.

Broadfoot, A. L., et al., Ultraviolet spectrometer observations of Uranus, Science, 233, 74, 1986.

Clarke, J. T., W. G. Fastie, P. D. Feldman, H. W. Moos, H. A. Weaver, and C. B. Opal, Spatial imaging of the Jupiter Lyman- $\alpha$ emission from rocket and IUE observations, Eos Trans. $A G U, 60$, 304, 1979.

Clarke, J. T., H. W. Moos, and P. D. Feldman, IUE monitoring of the spatial distribution of the H Ly $\alpha$ emission from Jupiter, Astrophys. J. Lett., 245, L127, 1981 a.

Clarke, J. T., H. W. Moos, S. K. Atreya, and A. L. Lane, IUE detection of bursts of H Ly $\alpha$ emission from Saturn, Nature, 290, 226, $1981 b$.

Clarke, J. T., H. W. Moos, and P. D. Feldman, The far-ultraviolet 
spectra and geometric albedos of Jupiter and Saturn, Astrophys. $J$., $255,806,1982$.

Clarke, J. T., et al., Continued observations of the H Ly a emission from Uranus, J. Geophys. Res., 91, 8771, 1986.

Connerney, J. E. P., M. H. Acuna, and N. F. Ness, The magnetic field of Uranus, J. Geophys. Res., this issue.

Cramer, W. H., Elastic and inelastic scattering of low-velocity $\mathrm{H}^{+}$and $\mathrm{H}_{2}{ }^{+}$in hydrogen, J. Chem. Phys., 35, 836, 1961.

Cravens, T. E., G. A. Victor, and A. Dalgarno, The absorption of energetic electrons by molecular hydrogen gas, Planet. Space Sci., $23,1059,1975$.

Dalgarno, A., M. B. McElroy, and A. I. Stewart, Electron impact excitation of the dayglow, J. Atmos. Sci., 26, 753, 1969.

Dessler, A. J., B. R. Sandel, and S. K. Atreya, The Jovian hydrogen bulge: Evidence for co-rotating magnetospheric convection, Planet. Space Sci., 29, 215, 1981.

Festou, M. C., S. K. Atreya, T. M. Donahue, B. R. Sandel, D. E. Shemansky, and A. L. Broadfoot, Composition and thermal profiles of the Jovian upper atmosphere determined by the Voyager ultraviolet stellar occultation experiment, J. Geophys. Res., 86, 5715, 1981.

Franck, J., and G. Hertz, Nobel Lectures in Physics 1922-1941, pp., 93-132, Elsevier, New York, 1965. (Also Verh. Phys. Ges., 16, 457 and 512,1914$)$.

Hanson, W. B., and R. J. Moffett, Ionization transport effects in the equatorial $F$ region, J. Geophys. Res., 71, 5559, 1966.

Hinteregger, H. E., K. Fukui, and B. R. Gibson, Observational reference and model data on solar EUV, from measurements on AE-E, Geophys. Res. Lett., 8, 1197, 1981.

Hunten, D. M., and A. J. Dessler, Soft electrons as a possible heat source for Jupiter's thermosphere, Planet. Space Sci., 25, 817, 1977.

Lee, M.-P., R. R. Lucchese, and E. McCoy, Electron-impact excitation and dissociation processes in $\mathrm{H}_{2}$, Phys. Rev. $A, 26,3240,1982$.

Mahajan, K. K., Equatorial anomaly in the Jovian ionosphere, Geophys. Res. Lett., 8, 66, 1981.

McConnell, J. C., B. R. Sandel, and A. L. Broadfoot, Airglow from Jupiter's nightside and crescent: Ultraviolet spectrometer observations from Voyager 2, Icarus, 43, 128, 1980.

Pfaff, R., Rocket studies of plasma turbulence in the equatorial electrojet, Ph.D. dissertation, Cornell University, Ithaca, N. Y., 1986.

Prange, R., New evidence for the role of photoelectrons in the $\mathrm{H}_{2}$-dayglow of the giant planets, Astron. Astrophys., 161, L1, 1986.

Rishbeth, H., The F-layer dynamo, Planet. Space Sci., 19, 263, 1971.

Rishbeth, H., and O. K. Garriott, Introduction to Ionospheric Physics, Academic, Orlando, Fla., 1969.

Sandel, B. R., et al., Extreme ultraviolet observations from Voyager 2 encounter with Jupiter, Science, 206, 962, 1979.
Sandel, B. R., A. L. Broadfoot, and D. F. Strobel, Discovery of a longitudinal asymmetry in the hydrogen Lyman-alpha brightness of Jupiter, Geophys. Res. Lett., 7, 5, 1980.

Shemansky, D. E., An explanation for the $H$ Ly $\alpha$ longitudinal asymmetry in the equatorial spectrum of Jupiter, J. Geophys. Res., 90, 2673,1985 .

Skinner, T. E., S. T. Durrance, P. D. Feldman, and H. W. Moos, Temporal variations of the Jovian H I Lyman-alpha emission, Astrophys. J. Lett., 265, L23, 1983.

Smith, B. A., et al., Voyager 2 in the Uranian system: Imaging science results, Science, 233, 43, 1987.

Smith, G. R., D. E. Shemansky, J. B. Holberg, A. L. Broadfoot, and B R. Sandel, Saturn's upper atmosphere from the Voyager 2 EUV solar and stellar occultations, J. Geophys. Res., 88, 8667, 1983.

Swift, D. W., A mechanism for energizing electrons in the magnetosphere, J. Geophys. Res., 70, 3061, 1965.

Tan, A., Spatial and diurnal features of the Jovian equatorial anomaly, Planet. Space Sci., 34, 117, 1986.

Trajmar, S., D. F. Register, and A. Chutjian, Electron scattering by molecules, II, Experimental methods and data, Phys. Rep., 97, 219, 1983.

Yelle, R. V., B. R. Sandel, D. E. Shemansky, and S. Kumar, Altitude variation of EUV emissions and evidence for proton precipitation at low latitudes in the Saturnian atmosphere, J. Geophys. Res., 9I, $8756,1986$.

Yelle, R. V., L. R. Doose, M. G. Tomasko, and D. F. Strobel, Analysis of Raman scattered Ly- $\alpha$ emissions from the atmosphere of Uranus, Geophys. Res. Lett., 14, 483, 1987.

Yung, Y. L., and D. F. Strobel, Hydrocarbon photochemistry and Lyman alpha albedo of Jupiter, Astrophys. J., 239, 395, 1980.

Yung, Y. L., G. R. Gladstone, K. M. Chang, J. M. Ajello, and S. K. Srivastava, $H_{2}$ fluorescence spectrum from 1200 to $1700 \AA$ by electron impact: Laboratory study and application to Jovian aurora, Astrophys. J. Lett., 254, L65, 1982.

J. T. Clarke, Department of Atmospheric and Oceanic Sciences, University of Michigan, Ann Arbor, MI 48109.

M. K. Hudson, Department of Physics and Astronomy, Dartmouth College, Hanover, NH 03755.

Y. L Yung, Division of Geologic and Planetary Sciences, California Institute of Technology, Pasadena, CA 91125.

(Received March 25, 1987;

revised October 5, 1987;

accepted October 9, 1987.) 